\title{
Clinical Response to Treatment of Central Nervous System Tuberculosis in Non-Human Immunodeficiency Virus-Infected Adolescents and Adults
}

\author{
Jin Young Lee1, Su Jin Lee ${ }^{2 *}$, Ji Young Park¹, Min Jeong Kim³ \\ ${ }^{1}$ Department of Internal Medicine, Kosin Medical College, Busan, Korea \\ ${ }^{2}$ Department of Internal Medicine, Pusan National University, Busan, Korea \\ ${ }^{3}$ Department of Neurology, Kosin Medical College, Busan, Korea \\ Email: rejim@hanmail.net, ^beauty192@hanmail.net, aiteite1@naver.com,merritt329@hanmail.net
}

How to cite this paper: Lee, J.Y., Lee, S.J., Park, J.Y. and Kim, M.J. (2016) Clinical Response to Treatment of Central Nervous System Tuberculosis in Non-Human Immunodeficiency Virus-Infected Adolescents and Adults. Journal of Tuberculosis Research, 4, 173-182.

http://dx.doi.org/10.4236/jtr.2016.44020

Received: July 8, 2016

Accepted: December 4, 2016

Published: December 7, 2016

Copyright $\odot 2016$ by authors and Scientific Research Publishing Inc. This work is licensed under the Creative Commons Attribution International License (CC BY 4.0).

http://creativecommons.org/licenses/by/4.0/ (c) (i) Open Access

\section{Abstract}

Introduction: More than half of patients with central nervous system tuberculosis (CNS TB) die or are left with severe neurological deficits despite receiving anti-TB treatment. Aims of the study: This study examined risk factors associated with poor response to initial treatment with four anti-TB drug regimens or three drug regimens with steroids as adjuvant therapy. Methods: This study analyzed medical records from two tertiary hospitals in Busan, Korea, between January 2009 and March 2012. The subjects were non-human immunodeficiency virus (HIV)-infected patients aged $\geq 16$ years with clinical CNS TB. The subjects were divided into two groups according to response to treatment. Results: In totally, 52 patients with CNS TB were included. Of these, $14(26 \%)$ and 38 (73\%) showed poor and good responses, respectively. Of the patients with poor response, nine had stage III disease (64.3\%) according to the British Medical Research Council (BMRC) staging system. A significantly higher proportion was seen in the good response group $(\mathrm{p}<0.05)$. Patients with positive cerebrospinal fluid (CSF) acid-fast bacillus (AFB) culture, positive sputum AFB culture, positive CSF TB polymerase chain reaction (PCR) results, and brain tuberculoma had poorer responses $(\mathrm{p}<0.05)$. Multivariate analysis to determine risk factors associated with poor response to anti-TB therapy revealed that a poor response was associated with stage III clinical signs upon diagnosis (odds ratio [OR] 32.122; 95\% confidence interval [CI] 2.221 - 464.605), positive sputum AFB culture (OR 13.624; 95\% CI 1.066 - 174.149), and tuberculoma on brain images (OR 45.714; 95\% CI 1.893 - 1104.018). Conclusions: The results demonstrate the importance of identifying the severity of CNS TB and promptly administering anti-TB drugs. It is necessary to 
perform drug susceptibility testing for anti-TB drugs. Further studies are needed to confirm the correlations between risk factors associated with poor response and antiTB drug resistance and the other risk factors.

\section{Keywords}

Central Nervous System Tuberculosis, Poor Response, Severity of Symptoms, Anti-Tuberculosis Drug Resistance

\section{Introduction}

Central nervous system tuberculosis (CNS TB) begins with hematogenous spread of Mycobacterium tuberculosis from the primary pulmonary TB site, and eventually leads to lesions in the brain and spinal cord. The timing and risk of developing symptoms related to primary respiratory infections are associated with age and immune status. Immunosuppressed adults, particularly those infected with human immunodeficiency virus (HIV), are at high risk for CNS TB. And other risk factors include diabetes, malignancy, alcoholism, steroid therapy, and use of tumor necrosis factor (TNF)-blocking agents [1] [2]. CNS TB causes death or severe neurologic disabilities in more than 50\% of the patients, despite administration of appropriate anti-TB drugs and adjuvant steroid therapies [3]. Early initiation of effective anti-TB therapy plays the most critical role in successful outcomes; however, many cases demonstrate a poor response even when anti-TB therapy is administered [4]. There were a few studies about tuberculosis related deaths and risk factors for treatment failure of tuberculosis. Fatality was related to older age, disseminated disease, co-morbidity and social and geographical isolation [5] [6]. But there was not study about factors including laboratory data and image findings associated with poor response to tuberculosis treatment. The present study aimed to determine the risk factors associated with poor response of non-HIV infected patients despite initial treatment with anti-TB drugs and corticosteroids.

\section{Materials and Methods}

\subsection{Study Area and Population}

The present study was conducted in two tertiary university-affiliated hospitals with more than 900 beds in Busan City, South Korea, from January 2009 to March 2012. The incidence of TB in Busan City is the highest in the nation, at 86.9 per 100,000 population (national average 72.4 ) in 2005, 95.5 per 100,000 population (national average 78.9) in 2011, and 93.6 per 100,000 population (national average 78.5) in 2012 [7]. A study investigating multidrug-resistant TB in one tertiary hospital in Busan from 2005 to 2009 , which did not participate in the present study, reported that 312 of 367 total subjects were new cases (85.0\%). In that study, 75 (20.4\%) of the 367 patients were resistant to at least one drug, and the prevalence of drug-resistant TB was significantly higher in the group of previously treated patients (44 [14.1\%] patients in the new case 
group vs. 31 [56.4\%] patients in the previously treated group; $\mathrm{p}<0.01$ ). Isoniazid (INH) resistance was most common (18.8\%), followed by that of rifampin (RFP, 10.9\%), ethambutol (EMB, 7.1\%), streptomycin (SM, 4.9\%), and quinolone (2.7\%) [8].

\subsection{Data Collection Techniques}

The present study was conducted retrospectively using medical records. The subjects were non-HIV-infected patients aged 16 years or older with CNS TB. The diagnosis of CNS TB was classified as confirmed or probable TB. Patients were defined as having confirmed disease if clinical cerebrospinal fluid (CSF) or sputum specimens were positive for M. tuberculosis on culture, acid-fast bacilli (AFB) staining, or M. tuberculosis polymerase chain reaction (PCR) assay. Patients were defined as having probable TB when chest radiography images showed active lesions, there was response to anti-TB therapy, or when CNS TB was clinically suspected. Patients were diagnosed at one of the two hospitals, and patients were excluded if they had serologic evidence of HIV infection, microbiological evidence of non-TB mycobacterial (NTM) infection, or incomplete medical records. Patients were also excluded if they showed a paradoxical response during the course of treatment. Paradoxical response was defined as initial improvement after receiving anti-TB drugs and steroid treatments for at least two weeks, followed by clinical or radiographic deterioration of lesions or development of new lesions. The demographic characteristics of the subjects included sex, age, history of previously treated tuberculous condition, classification of confirmed or probable diagnosis, and comorbid diseases (diabetes, chronic liver disease, malignancy, heart failure). Clinical signs were assessed according to the Modified British Medical Research Council (BMRC) staging system [4]. Symptoms such as headache for more than one week, nausea, vomiting, fever, altered consciousness, seizures, and neurologic abnormalities were examined. Laboratory assessments included CSF examination (opening pressure, leukocytes with differentiation, protein level, glucose ratio, lactate dehydrogenase [LDH] level, adenosine deaminase [ADA] level), serum leukocyte count, and c-reactive protein [CRP] level. Poor response to initial treatment was defined as clinical or radiographic deterioration without improvement for two months after initiation of treatment or development of new lesions without improvement within two months of treatment initiation. During these two months, TB culture and anti-TB drug susceptibility test results become identifiable; thus, response to initial treatment could be compared between patients treated with the same anti-TB drugs before switching drugs. The initial treatment began with a four-drug regimen of INH (5 mg/kg), RFP (10 mg/kg), EMB (25 mg/kg), and pyrazinamide (PZA, $15-20 \mathrm{mg} / \mathrm{kg}$ ) or a three-drug regimen (INH, RFP, EMB) plus dexamethasone $(0.4 \mathrm{mg} / \mathrm{kg} / 24$ hours). The presence of TB in non-CNS sites including the lungs, extrathoracic lymph nodes, skeletal system, heart, liver, and spleen was also examined.

\subsection{Analysis}

Statistical analysis was performed using IBM SPSS Statistics for Windows, version 23.0 
(IBM Corp., Armonk, NY, USA). Correlations between categorical variables were evaluated using Fisher's exact test, and continuous variables were evaluated using Student's $\mathrm{t}$-test. Independent predictors of poor response were analyzed using logistic regression analysis, and a p-value less than 0.05 was considered statistically significant.

\subsection{Ethics Statement}

This study was approved by the Institutional Review Board of Kosin University Gospel Hospital.

\section{Results}

\subsection{Demographic and Clinical Characteristics of Patients with Central Nervous System Tuberculosis}

During the 39-month study period, 52 patients with CNS TB were included in the analysis. Baseline clinical characteristics were compared in those with good or poor response to initial treatment. Of these 52 patients, 14 (26\%) showed a poor response, while $38(73 \%)$ showed a good response. The mean age ( \pm standard deviation) of all subjects was $46.1 \pm 17.4$ years. The mean age of those with a good response was $46.1 \pm$ 16.8 years, higher than the $45.9 \pm 19.6$ years for those in the poor response group, but the difference was not statistically significant $(\mathrm{p}=0.98)$. In terms of clinical symptoms that were evaluated upon diagnosis and initiation of treatment, in accordance with the Modified BMRC staging system, nine patients (64.3\%) in the poor response group had stage III disease, with a higher proportion of patients with severe clinical symptoms; this difference was statistically significant $(\mathrm{p}<0.05)$. When the presence or absence of TB in non-CNS sites such as the lungs, extrathoracic lymph nodes, skeletal system, heart, liver, and spleen in addition to CNS TB was examined, $2.1 \pm 0.6$ involved sites were identified in the good response group, which was significantly higher than that for the poor response group $(1.4 \pm 0.6 ; \mathrm{p}<0.05)$. Comorbidity with CNS TB was identified in 14 subjects, with the lungs being the most common site of involvement (nine subjects, $60 \%$ ). Of 14 subjects with a poor response, five had lung involvement, two of whom had miliary TB. The remaining sites of involvement were the extrapulmonary lymph nodes (four subjects, 26.6\%) and liver (one subject, 7.1\%). The study population consisted of 30 men $(57.29 \%)$ and 22 women (42.31\%). Poor response was significantly associated with female sex and confirmed diagnosis $(\mathrm{p}<0.05)$. In addition, no statistical significance was found between patients receiving four-drug or three-drug regimens for treatment of CNS TB. The mean treatment durations in the good and poor response groups were $14.8 \pm 6.4$ (median months $\pm \mathrm{SD}$ ) and $14.2 \pm 11.5$ months, respectively, a difference that was not statistically significant (Table 1 ).

\subsection{Laboratory Data and Brain Imaging of Patients with Central Nervous System Tuberculosis}

Comparative analysis for accompanying constitutional symptoms, underlying disease, CSF opening pressure, white blood cell (WBC) count, lymphocyte count, protein level, 
Table 1. Demographic and clinical characteristics of patients with central nervous system tuberculosis with good and poor response to initial treatment.

\begin{tabular}{|c|c|c|c|}
\hline & Good response $(\mathrm{n}=38)$ & Poor response $(\mathrm{n}=14)$ & p-value \\
\hline Age, mean years \pm SD & $46.1 \pm 16.8$ & $45.9 \pm 19.6$ & 0.98 \\
\hline Sex (Female), number (\%) & $12(31.6 \%)$ & $10(71.4 \%)$ & 0.01 \\
\hline Prior TB therapy & & & 0.003 \\
\hline None, number (\%) & $38(100 \%)$ & $11(78.6 \%)$ & \\
\hline Previous TB therapy, number (\%) & 0 & $3(21.4 \%)$ & \\
\hline Diagnosis & & & 0.00 \\
\hline Confirmed, number (\%) & $6(15.8 \%)$ & $10(71.4 \%)$ & \\
\hline Probable, number (\%) & $32(84.2 \%)$ & $4(28.6 \%)$ & \\
\hline BMRC severity grade ${ }^{\star}$ & & & 0.00 \\
\hline I, number $(\%)$ & $28(73.7 \%)$ & $2(14.3 \%)$ & \\
\hline II, number (\%) & $6(15.8 \%)$ & $3(21.4 \%)$ & \\
\hline III, number (\%) & $4(10.5 \%)$ & $9(64.3 \%)$ & \\
\hline Constitutional symptoms, mean \pm SD & $2.2 \pm 1.1$ & $2.2 \pm 0.9$ & 0.87 \\
\hline Headache, number (\%) & $32(84.2 \%)$ & $10(71.4 \%)$ & \\
\hline Nausea/vomiting, number (\%) & $16(42.1 \%)$ & $4(28.5 \%)$ & \\
\hline Fever, number (\%) & $18(47.3 \%)$ & $7(50.0 \%)$ & \\
\hline Loss of consciousness, number (\%) & $4(10.5 \%)$ & $9(64.2 \%)$ & \\
\hline Underlying disease, mean \pm SD & $0.5 \pm 0.8$ & $0.4 \pm 0.6$ & 0.48 \\
\hline DM, number (\%) & $11(28.9 \%)$ & $2(14.2 \%)$ & \\
\hline Heart failure, number (\%) & $2(5.2 \%)$ & $1(7.1 \%)$ & \\
\hline Chronic liver disease, number (\%) & $1(2.6 \%)$ & 0 & \\
\hline Malignancy, number (\%) & $1(2.6 \%)$ & $1(7.1 \%)$ & \\
\hline Comorbidity, mean \pm SD & $2.1 \pm 0.6$ & $1.4 \pm 0.6$ & 0.00 \\
\hline Lung, number (\%) & $4(10.5 \%)$ & $5(35.7 \%)$ & \\
\hline Extrapulmonary lymph node, number (\%) & $1(2.6 \%)$ & $3(21.4 \%)$ & \\
\hline Liver, number (\%) & 0 & $1(7.1 \%)$ & \\
\hline TB treatment regimen & & & 0.57 \\
\hline Four-drug & $30(78.9 \%)$ & $10(71.4 \%)$ & \\
\hline Three-drug & $8(21.1 \%)$ & $4(28.6 \%)$ & \\
\hline Duration of therapy, median months \pm SD & $14.8 \pm 6.4$ & $14.2 \pm 11.5$ & 0.84 \\
\hline
\end{tabular}

TB, tuberculosis; DM, diabetes mellitus. ${ }^{\star}$ The modified British Medical Research Council clinical criteria for tuberculous meningitis severity; Grade I, alert and orientated without focal neurological deficit; Grade II, Glasgow coma score 14 - 10 with or without focal neurological deficit or Glasgow coma score 15 with focal neurological deficit; Grade III, Glasgow coma score less than 10 with or without focal neurological deficit. 
glucose ratio, LDH level, and ADA level showed no statistical significance ( $p>0.05)$ between the two groups. Patients with positive CSF AFB culture, positive sputum AFB culture, positive CSF TB PCR assay findings and tuberculoma formation on brain imaging showed a poorer response compared with the remaining subjects $(\mathrm{p}<0.05)$. Patients without tuberculoma on brain imaging, those with basal meningeal enhancement or hydrocephalus, and normal patients were included in the comparison (Table 2).

\subsection{Final Outcomes of the 14 Patients with Poor Response}

The final outcomes of 14 patients with poor response included seven deaths (50.0\%), five cases of residual paresis (35.7\%), and two cases lost to follow-up (14.2\%) (Table 3).

\subsection{Risk Factors Associated with Poor Response to Initial Anti Tuberculosis Therapy}

In order to determine the risk factors associated with poor response to initial anti-TB therapy, multivariate analysis was performed using binary logistic regression analysis. The risk factors associated with poor response to initial anti-TB therapy included the stage of clinical signs upon diagnosis and initiation of treatment (stage III, odds ratio [OR] 32.122; 95\% CI 2.221 - 464.605), positive sputum AFB culture (OR 13.624; 95\% CI 1.066 - 174.149), and tuberculoma on brain images (odds ratio 45.714; 95\% CI 1.893 1104.018) (Table 4).

Table 2. Laboratory data and brain image findings of patients with central nervous system tuberculosis with good and poor response to initial treatment.

\begin{tabular}{lccc}
\hline & Good response $(\mathrm{n}=38)$ & Poor response $(\mathrm{n}=14)$ & p-value \\
\hline CSF AFB culture-positive, number (\%) & $2(5 \%)$ & $6(42 \%)$ & 0.001 \\
Sputum AFB culture-positive, number (\%) & $4(10.5 \%)$ & $10(71.4 \%)$ & 0.00 \\
CSF TB PCR-positive, number (\%) & $4(10.5 \%)$ & $7(50.0 \%)$ & 0.002 \\
CSF pressure, mean pr. mmHg $\pm \mathrm{SD}$ & $221 \pm 74$ & $241 \pm 136$ & 0.54 \\
CSF WBC, mean cells/ $\mu \mathrm{L} \pm \mathrm{SD}$ & $445 \pm 505$ & $229 \pm 360$ & 0.16 \\
CSF lymphocytes, mean cells/mL $\pm \mathrm{SD}$ & $81.1 \pm 20.6$ & $62.5 \pm 34.1$ & 0.08 \\
CSF protein, mean mg/dL $\pm \mathrm{SD}$ & $199 \pm 135$ & $378 \pm 482$ & 0.21 \\
CSF glucose/blood glucose ratio $\pm \mathrm{SD}$ & $0.39 \pm 0.11$ & $0.34 \pm 0.21$ & 0.45 \\
CSF LDH, mean amount IU/L $\pm \mathrm{SD}$ & $82.0 \pm 37.7$ & $323.3 \pm 677.9$ & 0.24 \\
CSF ADA, mean amount IU/L $\pm \mathrm{SD}$ & $8.7 \pm 6.2$ & $16.4 \pm 21.7$ & 0.28 \\
Serum WBC count, mean cells/ $\mu \mathrm{L} \pm \mathrm{SD}$ & $8654 \pm 3698$ & $7957 \pm 2904$ & 0.53 \\
Serum HS-CRP, mean amount mg/dL $\pm \mathrm{SD}$ & $3.0 \pm 5.1$ & $7.5 \pm 11.8$ & 0.21 \\
Brain image & & & 0.02 \\
Tuberculoma formation, number $(\%)$ & $2(5.3 \%)$ & $4(28.6 \%)$ & \\
Basal meningeal enhancement, number $(\%)$ & $12(31.5 \%)$ & $5(35.7 \%)$ & $2(14.2 \%)$ \\
Hydrocephalus, number $(\%)$ & $3(7.9 \%)$ & $3(21.4 \%)$ & \\
Normal, number $(\%)$ & $21(55.3 \%)$ & & \\
\hline
\end{tabular}

CSF, cerebrospinal fluid; AFB, acid-fast bacilli; PCR, polymerase chain reaction; ADA, adenosine deaminase activity. 
Table 3. Final outcomes of 14 patients with poor response.

\begin{tabular}{cc}
\hline Characteristics & Number (\%) of patients with poor response \\
\hline Death & $7(50.0 \%)$ \\
Residual paresis & $5(35.7 \%)$ \\
Loss to follow up & $2(14.2 \%)$ \\
\hline
\end{tabular}

Table 4. Factors associated with poor response to initial anti-tuberculosis therapy.

\begin{tabular}{ccccc}
\hline & OR $(95 \% \mathrm{CI})$ & p-value & AOR $(95 \% \mathrm{CI})$ & p-value \\
\hline Sex (female) & $5.42(1.409-20.816)$ & 0.01 & & \\
Diagnosis (confirmed) & $13.33(3.125-56.887)$ & 0.00 & & \\
Stage of clinical signs (III) & $15.30(3.393$ to 68.992$)$ & 0.00 & $32.122(2.221$ to 464.605$)$ & 0.01 \\
CSF AFB culture-positive & $13.50(2.290-79.592)$ & 0.001 & $11.664(0.683$ to 199.292$)$ & 0.09 \\
Sputum AFB culture-positive & $21.25(4.488-100.615)$ & 0.00 & $13.624(1.066$ to 174.149$)$ & 0.04 \\
CSF TB PCR-positive & $8.50(1.948-37.094)$ & 0.002 & & \\
Tuberculoma in brain image & $7.20(1.148-45.167)$ & 0.02 & $45.714(1.893$ to 1104.018$)$ & 0.01 \\
\hline
\end{tabular}

CSF, cerebrospinal fluid; AFB, acid-fast bacilli; TB, tuberculosis; PCR, polymerase chain reaction; OR, unadjusted odds ratio; AOR, adjusted odds ratio.

\subsection{Drug Susceptibility Testing}

This study was a retrospective analysis and anti-TB drug susceptibility was not assessed in all subjects at the time of acceptance of report on positive TB cultures. Drug susceptibility testing was conducted for only three (37\%) of eight patients with CSF culturepositive findings and five (35\%) of 14 patients with sputum AFB culture-positive findings. Of the eight conducted drug susceptibility testing, only one (12.5\%) was resistant to INH, seven were sensitive to all anti tuberculosis drugs including INH, RFP, EMB and PZA. This patient also showed a sputum AFB culture-positive result and died one month after diagnosis despite administration of a four-drug anti-TB regimen and steroid treatment.

\section{Discussion}

Anti-TB drug and steroid treatments increase survival rates of patients with CNS TB. In the present study, we examined factors associated with poor response despite administration of four-drug anti-TB regimens or three-drug anti-TB regimens with steroid treatment. Univariate analysis revealed that female, confirmed diagnosis, and CSF AFB culture-positive findings were associated with poor response, whereas these factors were not significantly associated in multivariate analysis.

The results of multivariate analysis revealed that stage III BMRC severity grade of clinical signs upon diagnosis, AFB culture-positive sputum, and tuberculoma on brain images were independent risk factors of poor response.

The results of the present study indicate that the most important factor affecting 
treatment outcomes such as mortality and neurologic sequelae was the disease stage based on the severity of clinical symptoms at diagnosis and treatment initiation. If treatment is initiated in stage I, patient mortality and morbidity rates are very low; if treatment is initiated in stage III, 50\% of patients with low Glasgow coma scores or neurological deficits die or are left with some degree of neurological deficit [9]. Therefore, when CNS TB is suspected, prompt diagnosis and treatment initiation for patients with mild disease state may be the most beneficial to ensure a good prognosis.

In addition, all patients included in the present study were treated equally with fouror three-drug regimens from the initiation of this study for two months. The outcomes were compared at two months; thus, anti-TB drug resistance may have also affected the outcomes.

The incidence of anti-TB drug resistance is reportedly lower in patients with extrapulmonary TB than in patients with pulmonary TB [10]; however, other studies have reported that the incidence of anti-TB drug resistance in patients with extrapulmonary TB is similar to that in patients with pulmonary TB [11]. A 2011 study on anti-TB drug resistance in patients with extrapulmonary $\mathrm{TB}$ conducted in a university hospital reported $8.9 \%$ and $1.8 \%$ incidences of resistance to at least one anti-TB drug and multidrug resistance, respectively [12]; these rates are similar or slightly lower than those for the total population of South Korea [13]. In our study, the incidence of drug resistance in patients who were tested for anti-TB drug resistance was found to $12.5 \%$; this rate is similar to that in South Korean patients with drug-resistant TB, but not to the incidence of multidrug resistance in patients with extrapulmonary TB [13]. Among firstline anti-TB drugs, INH plays the most important role in the treatment of TB meningitis owing to its rapid activity and excellent penetration into CSF [14]. Therefore, the presence or absence of INH resistance may affect patient outcomes. Correlations between the incidence of INH resistance and laboratory data and brain images should also be considered. For example, a study by Duo et al. found a relatively high incidence of $\mathrm{INH}$ resistance and poorer outcomes following anti-TB drug treatment in patients with TB meningitis who were CSF PCR-positive [15]. A study by Vinnard et al. reported that INH resistance was associated with mortality in patients with TB meningitis with $M$. tuberculosis-positive CSF cultures. The Center for Disease Control and Prevention states that INH resistance is a strong factor predictive of death in patients with TB meningitis [16]. Though, this study was a retrospective analysis, not all factors were controlled and anti-TB drug susceptibility was not assessed in all subjects. Because in this study anti TB drug susceptibility testing was conducted for only a few patients, the relation between anti-TB drug susceptibility and laboratory data could not be studied in detail. Therefore, further studies on anti-TB drug resistance in patients with CNS TB are needed. In this study, positive sputum AFB cultures and tuberculoma on brain images were also identified as risk factors of poorer outcomes in multivariate analysis in this study. In addition, disease outcome and prognosis may be affected by patient age, nutritional status, presence of miliary disease, and underlying diseases. However, the present study found no significant association with these variables, likely due to the 
small number of patients included in this study, which increased the potential for errors in interpreting the results of the analysis. Another limitation was the possibility of poor patient compliance. Because the present study was a retrospective study based on medical records, it was not possible to accurately assess differences in patient compliance to anti-TB drug treatment regimens.

\section{Conclusion}

Although CNS TB can have serious consequences, few studies have assessed outcomes or drug resistance in these patients or have compared them to those for patients with pulmonary TB. In areas where TB is prevalent, it is critical to identify severity in patients with CNS TB, according to consciousness and degree of neurological deficit, and to start prompt anti-TB drug treatment. In addition, specimens for microbiological diagnosis should be obtained and drug susceptibility testing for anti-TB drugs should be performed. It might also be necessary to switch drugs based on drug resistance determined while evaluating therapeutic response. CSF nucleic acid amplification tests should be conducted for all patients with suspected CNS TB, and subsequent molecular drug susceptibility tests may be useful for determining the prognosis of patients with positive findings. Based on the results of the present study, further studies are necessary to determine the associations between risk factors for therapeutic response and anti-TB drug resistance, as well as differences in outcomes according to baseline characteristics in patients with CNS TB.

\section{References}

[1] Bishburg, E., Sunderam, G., Reichman, L.B. and Kapila, R. (1986) Central Nervous System Tuberculosis with the Acquired Immunodeficiency Syndrome and Its Related Complex. Annals of Internal Medicine, 105, 210-213. https:/doi.org/10.7326/0003-4819-105-2-210

[2] Keane, J. (2005) TNF-Blocking Agents and Tuberculosis: New Drugs Illuminate an Old Topic. Rheumatology, 44, 714-720. https:/doi.org/10.1093/rheumatology/keh567

[3] Thwaites, G.E., Bang, N.D., Dung, N.H., Quy, H.T., Oanh, D.T.T., Thoa, N.T.C., et al. (2004) Dexamethasone for the Treatment of Tuberculous Meningitis in Adolescents and Adults. New England Journal of Medicine, 351, 1741-1751. https:/doi.org/10.1056/NEJMoa040573

[4] Thwaites, G.E. and Hien, T.T. (2005) Tuberculous Meningitis: Many Questions, Too Few Answers. The Lancet Neurology, 4, 160-170. https:/doi.org/10.1016/S1474-4422(05)70019-3

[5] Walpola, H.C., Siskind, V., Patel, A.M., Konstantinos, A. and Derhy, P. (2003) Tuberculosis-Related Deaths in Queensland, Australia, 1989-1998: Characteristics and Risk Factors. The International Journal of Tuberculosis and Lung Disease, 7, 742-750.

[6] Sterling, T.R., Zhao, Z., Khan, A., Chaisson, R.E., Schluger, N., Mangura B., et al. (2006) Mortality in a Large Tuberculosis Treatment Trial: Modifiable and Non-Modifiable Risk Factors. The International Journal of Tuberculosis and Lung Disease, 10, 542-549.

[7] Ko, W., Shon, H. and Lee, H. (2013) Annual Report on the Notified Tuberculosis in Korea 2014. Korea Centers for Disease Control and Prevention.

[8] Yoon, N.-B., Lee, S.-W., Park, S.-M., Jeong, I.-H., Park, S.-Y., Han, S.-Y., et al. (2011) The Current Status of Multidrug-Resistant Tuberculosis in One Tertiary Hospital in Busan, 
2005-2009. Tuberculosis and Respiratory Diseases, 71, 120-125.

https:/doi.org/10.4046/trd.2011.71.2.120

[9] Holdiness, M.R. (1990) Management of Tuberculosis Meningitis. Drugs, 39, 224-233. https:/doi.org/10.2165/00003495-199039020-00006

[10] Peto, H.M., Pratt, R.H., Harrington, T.A., LoBue, P.A. and Armstrong, L.R. (2009) Epidemiology of Extrapulmonary Tuberculosis in the United States, 1993-2006. Clinical Infectious Diseases, 49, 1350-1357. https:/doi.org/10.1086/605559

[11] Yang, H., Field, S., Fisher, D. and Cowie, R. (2005) Tuberculosis in Calgary, Canada, 19952002: Site of Disease and Drug Susceptibility. The International Journal of Tuberculosis and Lung Disease, 9, 288-293.

[12] Cho, O.-H., Park, K.-H., Park, S.Y., Moon, S.M., Chong, Y.P., Kim, M-N., et al. (2011) Drug-Resistant Extrapulmonary Tuberculosis. Infection \& Chemotherapy, 43, 258-261. https:/doi.org/10.3947/ic.2011.43.3.258

[13] Kim, H.J. (2011) Current Status of Tuberculosis in Korea. The Korean Journal of Medicine, 82, 257-262. https:/doi.org/10.3904/kjm.2012.82.3.257

[14] Thwaites, G.E., Lan, N.T.N., Dung, N.H., Quy, H.T., Thoa, N.T.C., Hien, N.Q., et al. (2005) Effect of Antituberculosis Drug Resistance on Response to Treatment and Outcome in Adults with Tuberculous Meningitis. Journal of Infectious Diseases, 192, 79-88. https:/doi.org/10.1086/430616

[15] Duo, L., Ying, B., Song, X., Lu, X., Ye, Y., Fan, H., et al. (2011) Molecular Profile of Drug Resistance in Tuberculous Meningitis from Southwest China. Clinical Infectious Diseases, 53, 1067-1073. https:/doi.org/10.1093/cid/cir663

[16] Vinnard, C., Winston, C.A., Wileyto, E.P., MacGregor, R.R. and Bisson, G.P. (2010) Isoniazid Resistance and Death in Patients with Tuberculous Meningitis: Retrospective Cohort Study. BMJ, 341, c4451. https:/doi.org/10.1136/bmj.c4451

Submit or recommend next manuscript to SCIRP and we will provide best service for you:

Accepting pre-submission inquiries through Email, Facebook, LinkedIn, Twitter, etc.

A wide selection of journals (inclusive of 9 subjects, more than 200 journals)

Providing 24-hour high-quality service

User-friendly online submission system

Fair and swift peer-review system

Efficient typesetting and proofreading procedure

Display of the result of downloads and visits, as well as the number of cited articles

Maximum dissemination of your research work

Submit your manuscript at: http://papersubmission.scirp.org/

Or contact jtr@scirp.org 